\title{
Constraining the orbit of the possible companion to $\beta$ Pictoris
}

\section{New deep imaging observations ${ }^{\star}$}

\author{
A.-M. Lagrange ${ }^{1}$, M. Kasper ${ }^{2}$, A. Boccaletti ${ }^{3}$, G. Chauvin ${ }^{1}$, D. Gratadour ${ }^{3}$, T. Fusco ${ }^{4}$, D. Ehrenreich ${ }^{1}$, D. Apai ${ }^{5}$, \\ D. Mouillet ${ }^{1}$, and D. Rouan ${ }^{3}$ \\ ${ }^{1}$ Laboratoire d'Astrophysique de l'Observatoire de Grenoble, Université Joseph Fourier, CNRS (UMR 5571), BP 53, \\ 38041 Grenoble, France, \\ e-mail: anne-marie.lagrange@obs.ujf-grenoble.fr \\ 2 European Southern Observatory, Karl Schwarzschild Straße, 2, 85748 Garching bei München, Germany \\ 3 Laboratoire d'Études Spatiales et d'Instrumentation en Astrophysique, Observatoire de Paris, CNRS (UMR 8109), \\ Université Pierre et Marie Curie, Université Paris-Diderot, 5 place Jules Janssen, 92195 Meudon, France \\ 4 Office National d'Études et de Recherches Aérospatiales, 29 avenue de la Division Leclerc, 92322 Châtillon, France \\ 5 Space Telescope Science Institute, 3700 San Martin Drive, Baltimore, MD 21218, USA
}

Received 18 March 2009/ Accepted 9 June 2009

\section{ABSTRACT}

Context. We recently reported on the detection of a possible planetary mass companion to $\beta$ Pictoris at a projected separation of $8 \mathrm{AU}$ from the star, using data taken in November 2003 with NaCo, the adaptive-optics system installed on the Very Large Telescope UT4. Even though no second epoch detection was available, there are strong arguments to favor a gravitationally bound companion rather than a background object. If confirmed and located at a physical separation of $8 \mathrm{AU}$, this companion would be the closest planet ever imaged, and above all, could have formed via core-accretion. Its apparent magnitude would indicate a typical temperature of $\sim 1500 \mathrm{~K}$ and a mass of $\sim 8 M_{\text {Jup }}$. Interestingly, a planet with such characteristics would explain the main morphological and dynamical peculiarities of the $\beta$ Pic system.

Aims. Our goal was to re-observe $\beta$ Pic five years later to again detect the companion or, in the case of a non-detection, constrain its orbit.

Methods. Deep adaptive-optics $L^{\prime}$-band direct images of $\beta$ Pic as well as $K_{\mathrm{s}}$-band Four Quadrant Phase Mask coronagraph images with were recorded with $\mathrm{NaCo}$.

Results. No point-like signal with the brightness of the companion candidate (apparent magnitudes $L^{\prime}=11.2$ or $K_{\mathrm{s}} \simeq 12.5$ ) was detected at projected distances down to $\simeq 6.5 \mathrm{AU}$ in the present data from the star (by comparison, the same limit was reached at $\simeq 5.5 \mathrm{AU}$ in the better quality November 2003 data). As expected, the non detection does not allow us to rule out a background companion from an observational point of view. We show that the non detection is consistent with orbital motion. Using these data and previous $K_{\mathrm{s}}$-band data obtained in 2004, we place strong constraints on the possible orbits of the companion.

Key words. instrumentation: adaptive optics - stars: early-type - stars: planetary systems - stars: individual: $\beta$ Pictoris

\section{Introduction}

The $\beta$ Pic disk of dust and gas has been regarded as the prototype of young (12-4 Myr, Zuckerman et al. 2001) planetary systems since the 1980 s and has revealed over the years an impressive amount of indirect signs pointing toward the presence of at least one giant planet.

Using $L^{\prime}$-band high-angular resolution imaging data obtained with the NAOS-CONICA adaptive optic system ( $\mathrm{NaCo})$ on the Very Large Telescope UT4, we discovered a point-like source with an apparent magnitude $L^{\prime}=11.2 \pm 0.3 \mathrm{mag}$ at $0{ }^{\prime} 411 \pm 0.008$ ( $~ 8$ AU) north-east of $\beta$ Pic, well aligned with the dust disk. Even though no second epoch data were available with enough sensitivity to detect the companion candidate (cc), we were confident that this signal was not due to a background object, as the associated probability was shown to be very low.

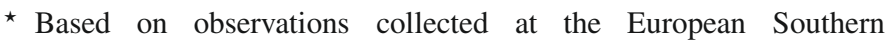
Observatory, Chile, ESO; runs 282.C5037(A), 282.C5037(B) and 282.C5037(D)
We therefore attributed the source to a probable bound object. Given the star proper motion, a background object would now lie angularly too close to the star to be detected again and a few more years (see below) are necessary to rule out this possibility from an observational point of view.

Very interestingly, if this $\mathrm{cc}$ is indeed at a physical separation of $8 \mathrm{AU}$, it would explain most of the $\beta$ Pic system's morphological and dynamical peculiarities: the disk inner warp, its brightness asymmetries, as well as the observed falling evaporating bodies (FEBs, Lagrange et al. 2009). Recently, Lecavelier des Etangs \& Vidal-Madjar (2009) investigated whether the observed cc could also be responsible for the photometric variability observed in November 1981 (Lecavelier des Etangs et al. 1995) and analysed in detail in a subsequent paper (Lecavelier des Etangs et al. 1997). In the latter paper, the complex photometric curve observed was explained either by a planet located in a dust-free region of the disk, or a cloud of dust passing in front of the star. In the planet scenario, an object with a radius larger than 2 Jupiter radii, 
located at less than $8 \mathrm{AU}$ and surrounded by a void of material within its Hill radius, could explain both the eclipse signal and the observed higher brightness a few days around the eclipse. From their dynamical analysis, Lecavelier des Etangs \& Vidal-Madjar (2009) conclude that the cc observed in 2003 could be responsible for the 1981 eclipse provided the semimajor axis of its orbit (circular case or assuming a low eccentricity) is in the range 7.6-8.7 $\mathrm{AU}$, corresponding to periods in the range 15.9-19.5 years. Another longer-period orbit, dynamically compatible with the existence of an eclipse and the November 2003 data, was excluded on the basis that the cc would have been detected earlier. We note that the corresponding orbital radius, $17 \mathrm{AU}$, is also much larger than the one predicted by the modeling of the photometric curve. As noted by these authors and Lagrange et al. (2009), a cc on an orbit with a radius of $\sim 8 \mathrm{AU}$ would not be detectable end of 2008 nor in early 2009. Lecavelier des Etangs \& Vidal-Madjar (2009) furthermore predict that if the $\mathrm{cc}$ is responsible for the 1981 eclipse, then it should reach its maximum elongation between 2011 and 2015 . Finally, we note that the data available to Lecavelier des Etangs \& Vidal-Madjar (2009) did not allow them to disentangle two possible cases: a first case where the cc would have been located before quadrature in 2003, and a second case where the cc would have been located after quadrature in 2003.

Obviously, new deep imaging observations are needed to further constrain the possible orbits of the cc. A major question is the true (unprojected) separation of the cc from the star. Four Quadrant Phase Mask (4QPM) coronagraph observations performed in 2004 did not reveal any point-like source with an absolute magnitude ${ }^{1}$ in the $K_{\mathrm{s}}$ band of $M_{K_{\mathrm{s}}} \simeq 11.5$ at a projected separation $>8 \mathrm{AU}$ (Boccaletti et al. 2009). Using the COND and DUSTY models (Baraffe et al. 2003; Chabrier et al. 2000), we obtained a $K_{\mathrm{S}}-L^{\prime}$ color of 1.2 (COND) to 1.4 (DUSTY); hence a cc with $L^{\prime}=11.2 \pm 0.3$ (i.e., an absolute magnitude $\left.M_{L^{\prime}}=9.8 \pm 0.3\right)$ would have $M_{K_{\mathrm{s}}}=11.0 \pm 0.3$ to $11.2 \pm 0.3$, respectively. If located beyond $8 \mathrm{AU}$, the cc seen in 2003 would then have been detected in these 4QPM data. This allows us to conclude that its projected separation had decreased between 2003 and 2004.

In order to confirm the companionship and/or to further constrain the cc orbit, we obtained discretionary time in January and February 2009 to perform new high-contrast and highspatial-resolution observations of the $\beta$ Pic system with $\mathrm{NaCo}$ (Rousset et al. 2003; Lenzen et al. 2003) in the $L^{\prime}$ band, thus allowing a direct comparison between 2003 and 2009 data, as well as with the $2004 \mathrm{~K}$ s-band 4QPM data ${ }^{2}$. The observations were designed to detect the faint companion as close as possible to the star. A positive detection would both confirm the companionship and provide crucial constraints on the companion orbital parameters. A non-detection would provide valuable constraints on the orbit of the cc. We present these observations in Sect. 2, and we use these new results to constrain the possible location of the cc in Sect. 3.

\footnotetext{
${ }^{1}$ In the following, absolute magnitudes in a photometric band $i$ are noted $M_{i}$ and calculated assuming a distance of $19.3 \mathrm{pc}$. The notation $i$ will simply refer to the apparent magnitude in this band.

2 Note that we additionally acquired images of $\beta$ Pic using the new Sparse Aperture Masking mode offered on NaCo in November 2008. However, the experimental observing template we tested as well as the SAM capabilites do not provide constraints on the cc. They are consequently not considered here.
}

\section{Observations and data reduction procedures}

\subsection{L'-band observations (February 2009)}

\subsubsection{Observing strategies}

$L^{\prime}$-band images of $\beta$ Pic $\left(V=3.8, L^{\prime}=3.5\right)$ were obtained in February 2009 with NaCo. Two different observing strategies were adopted. The first one (run A) followed as closely as possible the observing procedure adopted in November 2003, which consisted of recording $\beta$ Pic non-saturated and saturated images, followed by similar data on a comparison star, HR 2435 (also used in November 2003), whose saturated images are used to remove the stellar halo on the $\beta$ Pic saturated images.

For the second strategy (runs B1 and B2), $\beta$ Pic and HR 2435 images were recorded at two different de-rotator positions, to be able to use either $\beta$ Pic or HR 2435 to remove the star halo (see examples of the use of the star itself observed at different rotator positions to remove the PSF halo in Kasper et al. 2007). The offset between the two rotator positions was chosen to be $30^{\circ}$ (run B1) or $180^{\circ}$ (run B2). The latter was chosen such that the VLT aperture spiders remain in the same orientation.

For all runs, the time elapsed between $\beta$ Pic and HR 2435 observations was precisely calculated so that the images for both stars were recorded at similar parallactic angles, within $0.5^{\circ}$, so as to remove as accurately as possible the PSF wings.

\subsubsection{Instrumental set-up}

The visible wavefront sensor was used with the $14 \times 14$ lenslet array, together with the visible dichroic. We used a set-up similar to the one used for the November 2003 observations: the CONICA L27 camera, which provides a pixel scale of $\sim 27$ mas; note, however, that the CONICA detector was changed between observing runs. Saturated images of $\beta$ Pic were recorded, with detector integration times (DITs) of $0.2 \mathrm{~s}$ and a number of detector integrations (NDIT) of 150. For the saturated images, we used a four-position dithering pattern every two DIT $\times$ NDIT exposures. This allows accurate sky and instrumental background removal.

Non-saturated images were also recorded to obtain images of the stellar point spread function (PSF) as well as a photometric calibration. In such cases, we added the Long Neutral Density filter (transmission 0.018) in the CONICA optical path, and recorded images with DITs of $0.2 \mathrm{~s}$. Twilight flat fields were recorded as well.

The log of the observations is reported in Table 1, as well as the observing conditions. The observing conditions were noticeably not as good as during the November 2003 run, with coherent energies (estimated in the $K$ band) of $40-50 \%$ for run A, and $25-30 \%$ for runs B1 and B2, instead of $50-70 \%$ in 2003 , and coherent times $\tau_{0}$ between 2.8 and $6.2 \mathrm{~ms}$, instead of $20 \mathrm{~ms}$ in the best data sets in November 2003. The conditions during run $\mathrm{B} 1$ and even more during run $\mathrm{B} 2$ were rather poor, resulting in an unstable and mediocre adaptive optics (AO) correction.

\subsubsection{Data processing}

The data were reduced using different methods. The first two methods are described in Lagrange et al. (2009). A third method, which makes use of the different rotator positions in order to remove the $\beta$ Pic PSF halo, consists of the following steps. The initial steps of the image processing are standard (flat fielding, bad-pixel cleaning, sky subtraction). As in the previous methods, the sky for each exposure was determined using the exposure of 
Table 1. Observing log of the $\beta$ Pic saturated images, and corresponding atmospheric conditions.

\begin{tabular}{llllllllll}
\hline \hline Set & Star & Date & Filter & $\begin{array}{l}\text { DIT } \\
(\mathrm{s})\end{array}$ & NDIT & $\begin{array}{l}N_{\exp } \\
(\mathrm{s})\end{array}$ & $\begin{array}{l}\pi^{1} \\
(\mathrm{o})\end{array}$ & $\begin{array}{l}\langle E C\rangle^{2} \\
(\%)\end{array}$ & $\begin{array}{l}\left\langle\tau_{0}\right\rangle^{3} \\
(\mathrm{~ms})\end{array}$ \\
\hline A & $\beta$ Pic & $2009-02-11$ & $L^{\prime}$ & 0.2 & 150 & 48 & $-24.2 /-9.1$ & 43.4 & 5.0 \\
& HR 2435 & $2009-02-11$ & $L^{\prime}$ & $0.2 / 0.22$ & 150 & 48 & $-24.7 /-10.4$ & 49.0 & 4.9 \\
\hline B1 & $\beta$ Pic & $2009-02-16$ & $L^{\prime}$ & 0.2 & 150 & 44 & $-22.8 /-8.4$ & 35.7 & 3.2 \\
& HR 2435 & $2009-02-16$ & $L^{\prime}$ & 0.2 & 150 & 54 & $-22.9 /-6.7$ & 26.9 & 2.8 \\
\hline B2 & $\beta$ Pic & $2009-02-17$ & $L^{\prime}$ & 0.2 & 150 & 42 & $-16.8 /-2.4$ & 26.8 & 4.4 \\
& HR 2435 & $2009-02-17$ & $L^{\prime}$ & 0.2 & 150 & 42 & $-16.9 /-2.6$ & 28.0 & 6.2 \\
\hline D & $\beta$ Pic & $2009-01-20$ & $K_{\mathrm{s}}$ & 0.3 & 200 & 8 & $-30.9 /-21.5$ & 51.2 & 9.6 \\
& HR 2435 & $2009-01-20$ & $K_{\mathrm{s}}$ & 0.3 & 200 & 10 & $-31.3 /-18.2$ & 44.2 & 8.7 \\
\hline
\end{tabular}

1 Range of parallactic angles at the start/end of the observation.

2 The average coherent energy ( $K$-band) as estimated on-line by $\mathrm{AO}$ during saturated exposures.

3 The average coherence time as estimated on-line by AO during saturated exposures.

the next adjacent dithering position. Hence, the sky measurement for each exposure is never older than about half a minute. Then, the images were superimposed with sub-pixel accuracy by a cross-correlation technique and added, flux normalized to unit peak intensity using the flux in the unsaturated Airy rings and high-pass filtered. The high-pass filtering was done by subtracting from the image its median filtered $(\approx 3$ full-width-athalf-maximum box-width) version. This procedure efficiently eliminates the large-scale structures while leaving point sources (such as substellar companions) relatively unaffected. The highpass filtering reduces the PSF peak intensity by only about $15 \%$. Finally, images taken at different roll angles of the instrument were superimposed and subtracted from each other in order to calibrate residual speckle noise.

\section{2. $K_{\mathrm{s}}$-band observations (January 2009)}

\subsubsection{Observing strategy and instrumental set-up}

As the brightness ratio of the cc in $K_{\mathrm{s}}$ is expected to be larger than in $L^{\prime}$, a dedicated observing procedure was considered. Coronagraphic images of $\beta$ Pic were recorded in the $K_{\mathrm{s}}$ band with the 4QPM installed in 2007 in the $\mathrm{NaCo}$ focal wheel (Boccaletti et al. 2007) in combination with the Full Undersized pupil stop (10\% undersizing of the UT pupil). Additionally, a reference star was observed and used to calibrate the residual speckle halo (see below). Two series of exposures with DIT $=0.3 \mathrm{~s}$ and NDIT $=200$ were obtained at 4 different rotator positions: $0,15,30$ and $45^{\circ}$ clockwise, totaling $480 \mathrm{~s}$. Photometric references obtained on out-of-mask unsaturated images $($ DIT $=0.8 \mathrm{~s}$, NDIT $=20)$ are corrected for the Neutral Density transmission (1/90) and the difference of pupil stop (Boccaletti et al. 2008). The same observing procedure was repeated on the reference star HR 2435. However, the parallactic angles differs by $0.5,1.4,3.5$ and $3.3^{\circ}$ at each rotator angle. Differences of more than $0.5^{\circ}$ are not well adapted to our needs.

For these $K_{\mathrm{s}}$-band observations, the visible wavefront sensor again was used with the $14 \times 14$ lenslet array, together with the visible dichroic. We used the CONICA S13 camera, which provides a pixel scale of 13.25 mas. The DCS detector mode and readout modes were respectively set to "HighDynamic" and "Double".

\subsubsection{Data processing}

After standard flat-field correction and bad pixel removal, the 400 images $($ DIT $=0.3 \mathrm{~s}$ ) obtained at each rotator angle were co-added, resulting in 8 images of $120 \mathrm{~s}$ for the target star and similarly for the reference star. The stability of the stellar image behind the 4QPM was sufficient and did not cause significant variations of the coronagraphic attenuation, so image selection was not necessary. In addition to twilight flat field calibration, we acquired background observations with the 4QPM in the beam that we used to evaluate the 4QPM flat field alone. Correction of this flat field was made on the $120 \mathrm{~s}$ exposures as the subtraction of the IR background evaluated in the histogram of pixels. Images were recentered at the sub-pixel scale using the maximum of the cross-correlation map (we checked that it provides satisfying results on coronagraphic images). Then, several methods were considered to combine this set of images.

- Method 1: images are de-rotated and co-added, resulting in 2 images, one for the target star and one for the reference star. The speckle background is averaged by a factor equal to the number of rotator angles $\left(N_{\text {rot }}\right)$. An optimal subtraction is performed between the star and the reference.

- Method 2: image at rotator position $i+1$ is subtracted from image at position $i$. The resulting subtraction is de-rotated and co-added to the previous one. The same procedure is repeated on the reference star images. Finally, the reference image is subtracted from the target image. The speckle background here is averaged by a factor $N_{\text {rot }}-1$.

- Method 3: the target star image is subtracted from the reference star image for each rotator angle. As in method 2, this image at position $i+1$ is subtracted to image at position $i$. The final image is obtained after de-rotating and co-adding each of these subtractions. Again, the speckle are averaged by a factor $N_{\text {rot }}-1$.

- Method 4: the last method is closer to that of Marois et al. (2006). A median map is estimated on each pixel for the target set of images as for the reference star. This median map is subtracted from each rotator position. Then, the images are derotated and co-added. As for method 1, the background is averaged by a factor $N_{\text {rot }}$. 

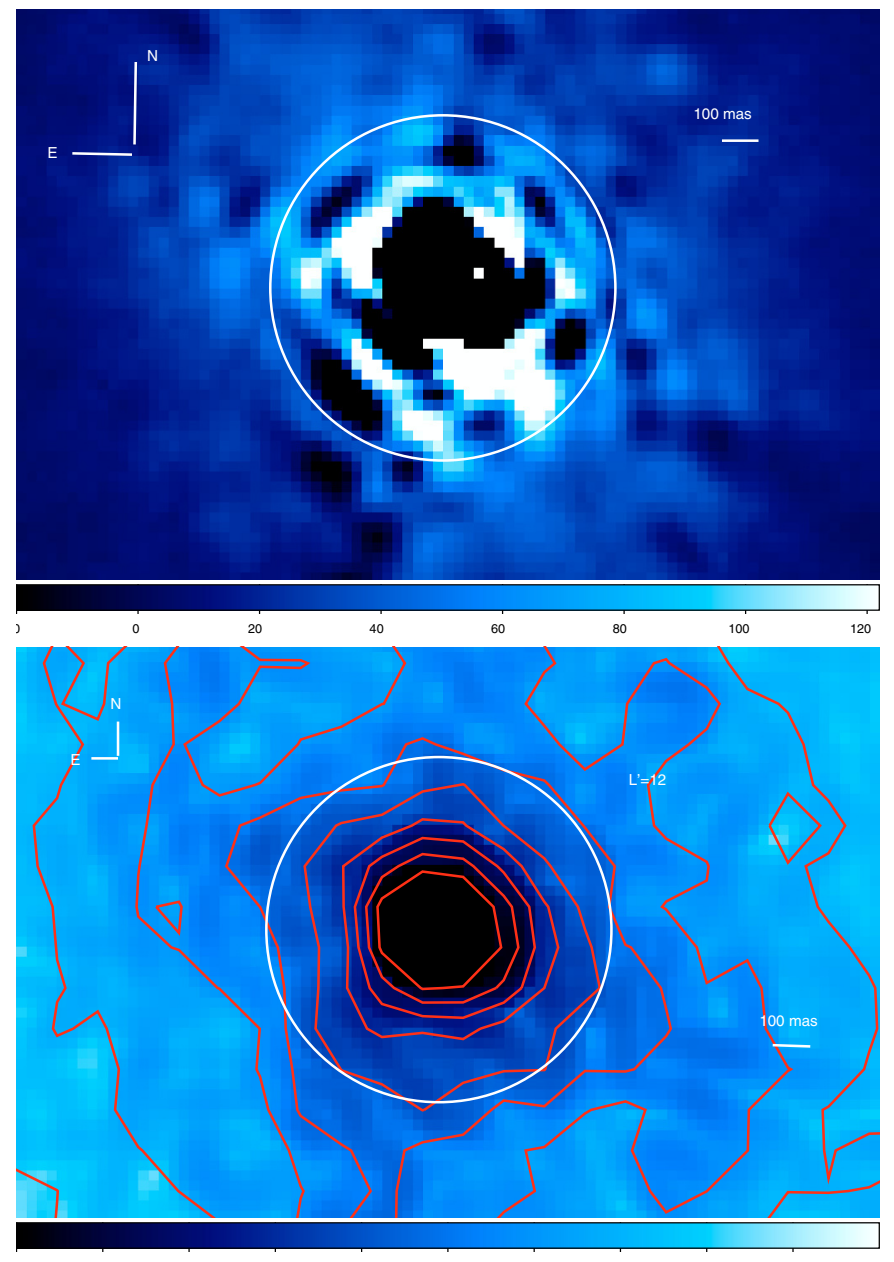

Fig. 1. Top: residual $L^{\prime}$ image after subtracting $\beta$ Pic from the comparison star HR 2435 (run A data). Bottom: map of the corresponding $6 \sigma$ detection limit. The contour levels represent iso-absolute magnitudes and are separated by $1 \mathrm{mag}$. The $M_{L^{\prime}}=12$ contour level is indicated for reference. The cc, with an absolute magnitude $M_{L^{\prime}} \simeq 9.8$ is not detected in these data. The radius of the white circle corresponds to $8 \mathrm{AU}$, i.e., the projected separation of the cc in November 2003.

Every time a subtraction is made between 2 images, we searched for the intensity ratio for an optimal subtraction by scanning a large range of possible ratios. This intensity ratio is then estimated in different ways (in the whole image, in a disk or in a ring) and the average is taken as the result. In principle, methods 2 and 3 are more efficient than traditional averaging of derotated images, as is shown in the $\mathrm{NaCo}$ user manual. However, in the present case, $N_{\text {rot }}=4$ is too small and so the gain of these methods is negligible. The results presented in this paper are obtained with method 4, although the performance of all the methods are not very different at an angular distance of 0.4 .

\section{Results}

\subsection{Detection limits}

We show in Fig. 1 the subtracted image corresponding to run A data, as well as the corresponding $6 \sigma$ detection limit obtained when using HR 2435 to remove the PSF halo. The detection limits were computed by measuring for each pixel the noise within a $5 \times 5$-pixel box centered on a given pixel, and determining the corresponding $6 \sigma$ limits, using the non-saturated images to obtain a photometric scaling factor, taking into account the instrumental set up used to record the non-saturated and saturated images.

No companion with $M_{L^{\prime}}=9.8$, corresponding to the absolute magnitude of the cc detected in November 2003 (if bound) is detected on the data down to a separation $r \simeq 11-13$ pixels, i.e., about 6.5 AU. Comparatively, $6 \sigma$ detection limits of $M_{L^{\prime}}=9.8$ were achieved down to $r=10-11$ pixels (i.e., about $5.5 \mathrm{AU}$ ) in the best set (set A) of the better quality November 2003 data. Taking into account the error bars associated with the measured magnitude $(0.3 \mathrm{mag})$ does not significantly change these results (the slope $\mathrm{d} r / \mathrm{d} M \simeq 4$ pixels mag $^{-1}$ at $r \simeq 12$ ).

Much less homogeneous results were obtained with run B1 data. Again using HR 2435 to remove the PSF halo, a $6 \sigma$ detection limits of $M_{L^{\prime}}=9.8$ is achieved down to $r=12-17$ pixels, depending on the position angle. This is coherent with the lower quality of the data compared to run $\mathrm{A}$ data. Using $\beta$ Pic as a comparison did not improve the results. Finally, run B2 data did not allow us to properly remove the PSF halo, as the image quality had changed signficantly between the recording of $\beta$ Pic and HR 2435 images. So, no attempt was made to derive detection limits.

Similarly, we show in Fig. 2 the results obtained in the $K_{\mathrm{s}}$ band. No companion is detected in the present data, which reach a typical limit of $M_{K_{\mathrm{s}}}=9$ at a separation of 0.4 (8 AU). An absolute magnitude $M_{K_{\mathrm{s}}}$ of 11.3 , corresponding to the one expected from the cc if bound (see above) is reached further than 40-44 pixels, i.e., at about $10 \mathrm{AU}$. Hence, the present $K_{\mathrm{s}}$ data do not allow us to test the presence of the cc at 0.4 (or less). For comparison, the computation of the $6 \sigma$ detection limit in the November $20044 \mathrm{QPM}$ data show that a cc with $M_{K_{\mathrm{s}}}=11.3$ would have been detected down to about 6.5-7 AU.

\section{Constraints on the physical position of the companion candidate}

\subsection{Constraints from available deep images}

First, we recall that as expected, the non-detection of the cc in February 2009 does not bring new constraints to the background object scenario. This can be seen in Fig. 3 where we have plotted the position of the cc between 2003 and 2013, taking into account the stellar proper motion. Note that we assume, as is usually done, that the cc has no proper motion). Should the object be a physical companion, our new non-detection would place strong constraints on its possible orbits; in the following we discuss this scenario.

We will assume that the $M_{L^{\prime}}=9.8 \mathrm{cc}$ orbits within the plane of the debris disk itself; the orbit is then seen edge-on as well; we furthermore assume that its orbit is prograde relative to the disk (see, e.g., Olofsson et al. 2001), and circular. Assuming a circular orbit is justified by the fact that the models proposed so far to explain the $\beta$ Pic disk peculiarities (disk asymmetries, FEBs) require planets on circular or low eccentric orbits (i.e., $\leq 0.1$; see the review in Lecavelier des Etangs \& Vidal-Madjar 2009), and as shown by the same authors, assuming such a low eccentricity does not significantly change the dynamical results. Besides, the relevant parameters adopted for the star itself are its mass, $1.75 \pm 0.05 M_{\odot}$, and its distance $19.3 \pm 0.2 \mathrm{pc}$ (Crifo et al. 1997).

In Fig. 4, we show the various regions of the $\beta$ Pic disk that were explored in November 2003 and/or in 2009. With only a single epoch of observation, an important part of the star's immediate surroundings is not explored. The new observations, in contrast, allow us to significantly increase the explored 

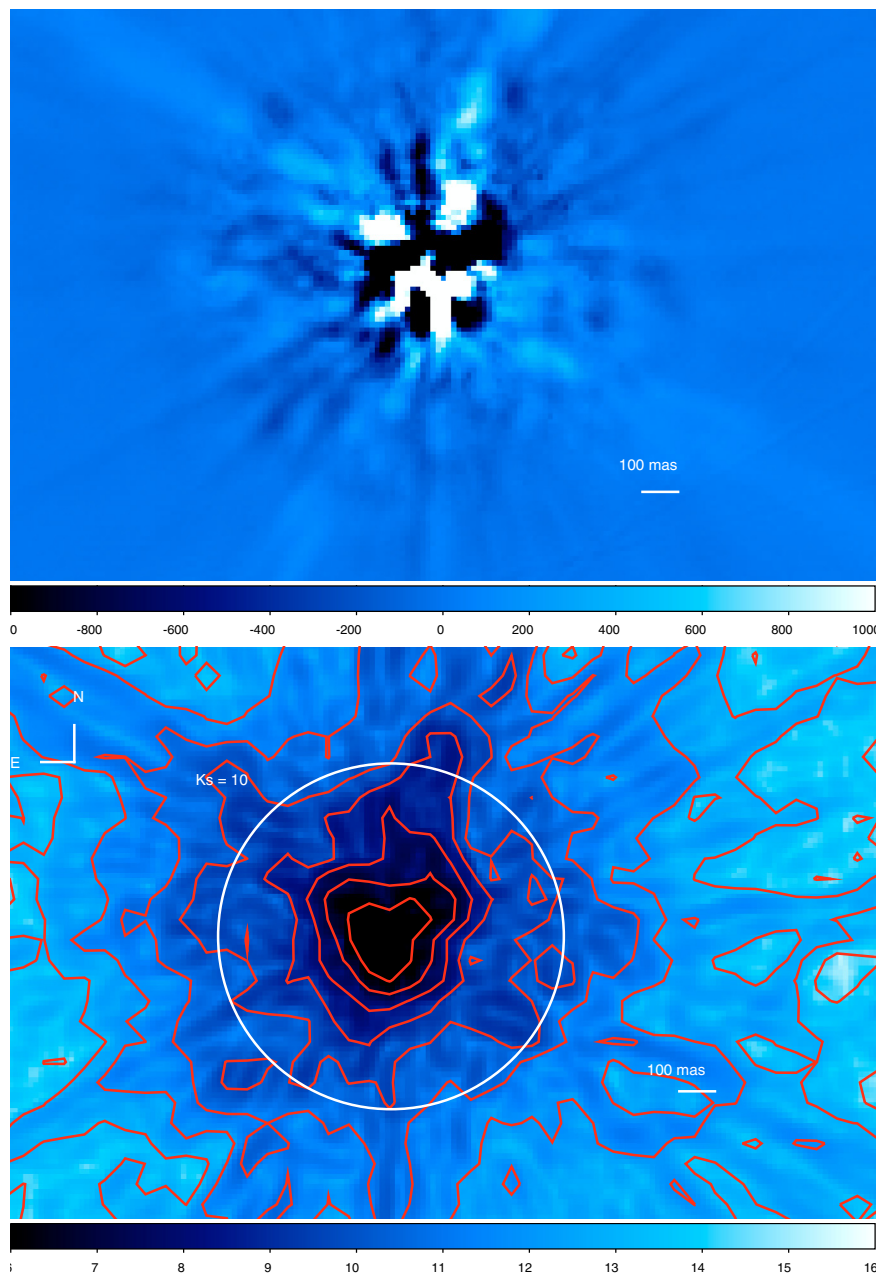

Fig. 2. Top: residual $K_{\mathrm{s}}$ image after subtracting $\beta$ Pic from the comparison star HR 2435. Bottom: map of the corresponding $6 \sigma$ detection limit. The contour levels represent iso-absolute magnitudes and are separated by $1 \mathrm{mag}$. The $M_{K_{\mathrm{s}}}=10$ contour level is indicated for reference. The cc with an expected absolute magnitude $M_{K_{\mathrm{s}}} \simeq 11.5$ is not detected in these data. North is up and east is to the left. The radius of the white circle corresponds to $8 \mathrm{AU}$, i.e., the projected separation of the $\mathrm{cc}$ in November 2003.

surroundings. We see, in particular, that the full 8-10 AU annulus around the star has now been fully explored, i.e., a companion with a mass higher than a few Jovian masses with a separation in the 8-10 AU range would have been detected either in 2003 or 2009 (circular orbit assumed). In other words, we can now exclude the presence of a massive $\mathrm{cc}$ at these separations, apart from the one detected in 2003.

We now constrain the physical position of the cc knowing that its projected separation was $8 \mathrm{AU}$ in November 2003 and is less than 6.5 AU in February 2009. In Fig. 5, we plot its projected separation in February 2009, assuming its projected separation was 8 AU on November 2003, as a function of its orbital radius. We note that the impact of the error associated with the star mass is negligible $(\leq 3 \%)$. We can see that:

- initial configurations where the cc orbited before quadrature (i.e., before the maximum elongation, see Fig. 7 for an illustration) in 2003 are ruled out except for separations between 8 and 9.75 AU. Larger separations are excluded. We note that to rule out a cc before quadrature and with a separation between 8 and $9.5 \mathrm{AU}$ with the $L^{\prime}$ data, we will need to wait until 2012-2013;

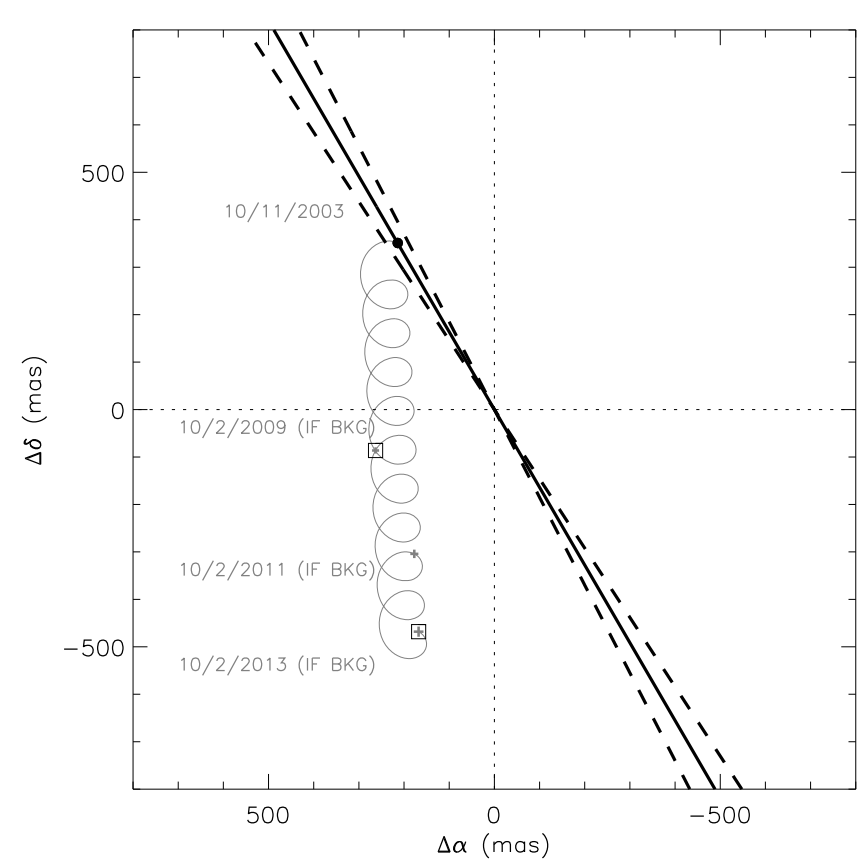

Fig. 3. Expected position of the source detected in November 2003 (black dot), assuming it was a background (BKG) object, taking the parallactic and stellar proper motions into account, for the 10 following years (thin curved line). Note that we assume, as is usually done, that the $\mathrm{cc}$ has no proper motion. The $\beta$ Pic debris disk midplane is indicated by the thick plain line. The thick dashed lines represent the uncertainty on the midplane angle.

- initial configurations where the cc orbited in 2003 after quadrature lead to current positions too close to the star to be detectable in February 2009. However, we note that for orbits with radii between 8 and $10 \mathrm{AU}$, the projected separation is about $4 \mathrm{AU}$ ( $\simeq 8$ pixels), so not far from the detection limits in the case of very good atmospheric conditions.

If we now take into account the non-detection of a cc with $M_{K_{\mathrm{s}}}=11.3$ down to 6.5-7.0 AU in the 4QPM November 2004 data, we see (Fig. 5) that (1) any cc orbiting before quadrature in 2003 would have been easily detected in 2004; (2) a cc orbiting after quadrature in 2003 with an orbital radius larger than $100 \mathrm{AU}$ would also have had in 2004 a separation large enough to be easily detected (even assuming the largest possible magnitude for the cc given the error bars, i.e., $M_{K_{\mathrm{s}}}=11.5$ ); (3) a cc orbiting after quadrature in 2003 with an orbital radius in the range 8.5 to $30 \mathrm{AU}$ would definitely not have been detected in the 4QPM 2004 data; and (4) a cc orbiting after quadrature in 2003 with an orbital radius in the range $30-100 \mathrm{AU}$ or smaller than about 8.5 AU would have had a separation in the range 6.5-7.1 AU in November 2004; given the uncertainties on both the $K_{\mathrm{s}}$ photometry and the uncertainty associated with the detection limits themselves, we cannot claim that the cc would have been detected in 2004.

We conclude then that the 2004 data definitely rule out any cc that would have been before quadrature in 2003, as well as a cc after quadrature in 2003, with an orbital radius larger than $100 \mathrm{AU}$. The data do not allow us to derive conclusions for $\mathrm{cc}$ orbital radii in the range 8 to $30-100$ AU. The conclusions derived from the 4QPM data rely on $K_{\mathrm{s}}-L^{\prime}$ color estimations. Knowing the actual $K_{\mathrm{s}}$ magnitude of the cc would therefore be very important. 


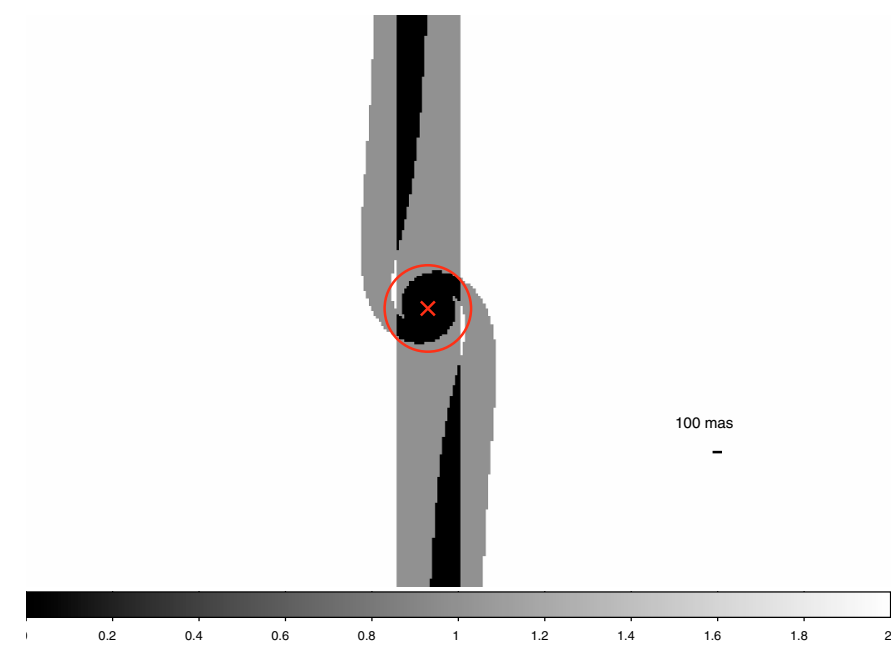

Fig. 4. Coverage map of the $\beta$ Pic system as seen from above (poleon). Regions (as seen in November 2003) which correspond to detection limits enough to detect the cc and which have been visited both in 2003 and 2009 (white area), once, ie either in 2003 or in 2009 (grey) and which have not been visited yet (black). $\beta$ Pic is indicated by a star. The circle represents an orbital radius of $8 \mathrm{AU}$ centered on the star. The disk rotates clockwise and the cc was $8 \mathrm{AU}$ on the left side of the star in November 2003 data. The two vertical lines correspond to separations of -6.5 and 6.5 AU. As a comparison, with the February 2009 (resp. November 2003) data only, the whole region "in front of" and "behind" the star at separations smaller than 6.5 AU (resp. 5.5 AU) were totally un-explored. Observing at two different epochs has allowed us to explore most of this region as well, thanks to the Keplerian rotation.

\subsection{Can the companion candidate still be responsible for the 1981 photometric eclipse?}

Lecavelier des Etangs \& Vidal-Madjar (2009) proposed that in order to be responsible for the 1981 eclipse, the cc observed in 2003 should have a physical separation in the range 7.6-8.7 AU (see Sect. 1). As underlined, they could not at that time disentangle a cc that was before or after quadrature in 2003. There was thus a degeneracy in the solutions.

We now test whether the constraints brought by the $2004 K_{\mathrm{S}}$ and $2009 L^{\prime}$ images still allow the cc to be the transiting planet of 1981. The 2004 data now excludes any cc that would have been before quadrature in 2003. This removes the degeneracy for the possible locations of the cc in November 2003. Assuming that the cc was at a projected separation of 8 AU in November 2003, and was after quadrature, we plot in Fig. 6 the positions of the cc in 1981. Three possible orbital radii are compatible, with a projected separation of 0 AU in 1981: 8.1, 10.5 and 17 AU. The radii at $10.5 \mathrm{AU}$ corresponds to an anti-transit solution (where the $\mathrm{cc}$ is aligned with the star and the observer, but is located exactly behind the star), so are not acceptable. The 8.1 and $17 \mathrm{AU}$ orbital radii correspond to transit positions in 1981 and therefore are dynamically acceptable.

An alternative (but equivalent) approach, as adopted by Lecavelier des Etangs \& Vidal-Madjar (2009) is to consider the projected separation of the cc in 2003 and 2009, assuming that it was transiting in 1981. In that case, we compute the projected separation assuming that at a time $t=0$, the $\mathrm{cc}$ is transiting, where as in the previous approach, we assumed that at $t=0$, the $\mathrm{cc}$ had a projected separation of $8 \mathrm{AU}$. The results are shown in Fig. 7. We see that the projected separation of the cc in 2003 is compatible with the detected (projected) position at $8 \mathrm{AU}$ for a semi-major axis of 8.1, 8.5, and 17.1 AU. The orbits with a
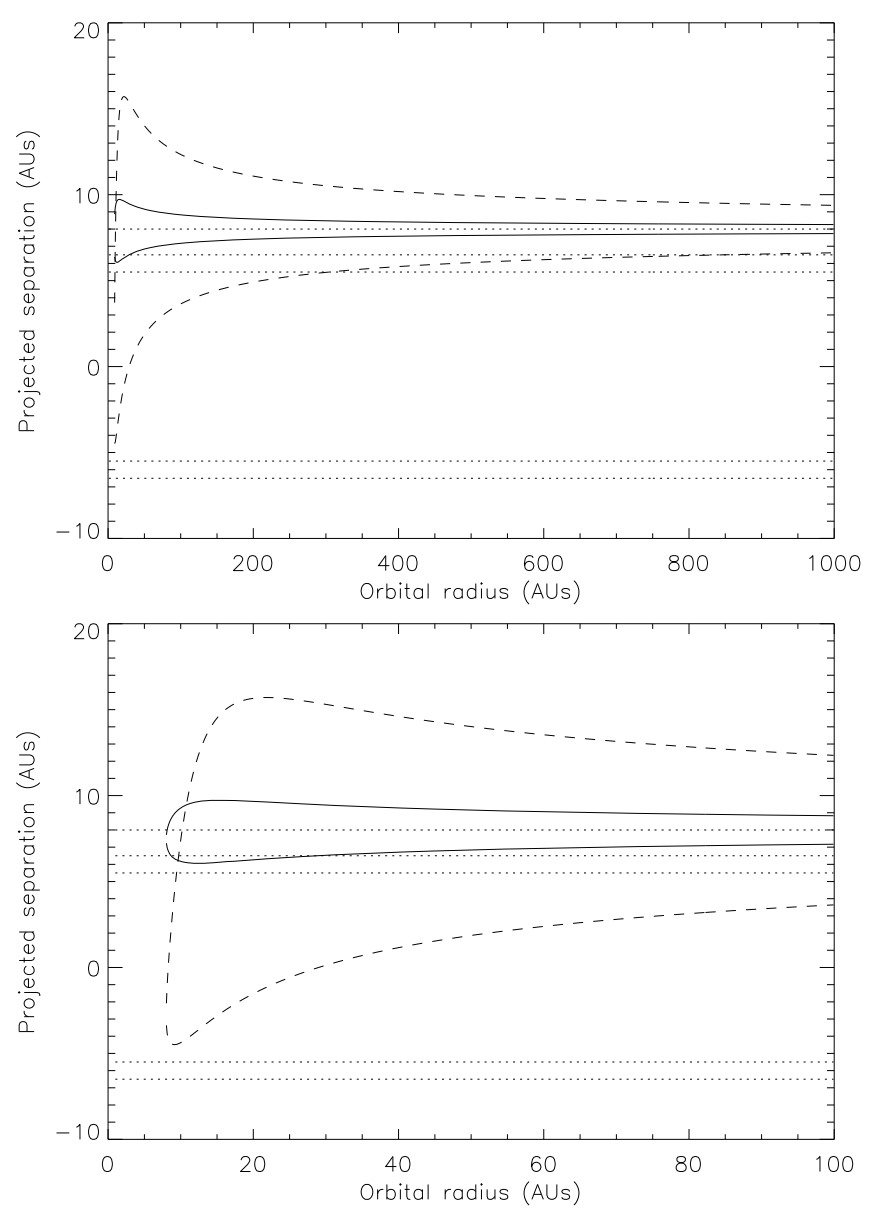

Fig. 5. Projected separations of the cc in 2009 (dashed curve) and 2004 (solid curve), as a function of its orbital radius, assuming its projected separation was $8 \mathrm{AU}$ in 2003. Top: orbital radii up to $1000 \mathrm{AU}$ are considered. Bottom: zoom of the same plot, with orbital radii up to $100 \mathrm{AU}$. The horizontal dotted lines indicate separations corresponding to the computed 6 sigma detection limits of an $M_{L^{\prime}}=9.8$ source in November 2003 (5.5 AU), and to an $M_{L^{\prime}}=9.8$ source in February 2009 (6.5 AU) or a $M_{K \mathrm{~s}}=11.3$ source in November 2004 (6.5 AU), and the cc projected separation in November 2003 (8 AU). Circular orbits are assumed and the $\mathrm{cc}$ is assumed to rotate prograde with respect to the circumstellar disk.

semi-major axis of 8.1 and $8.5 \mathrm{AU}$ correspond to positions before and after quadrature, respectively. The before-quadrature scenario is excluded by the 2004 4QPM images (see Fig. 4, right panel). We furthermore see that with the 8.1, and 17.1 AU solutions, the projected separation of the cc in February 2009 is much smaller than 6.5 AU (unhatched region in the top panel of Fig. 7). We conclude then that both possible semi-major axe of 8.5 and 17.1 AU are compatible with the cc being responsible for the 1981 eclipse, and being at a projected separation of 8 AU after quadrature in November 2003, and being undetected in February 2009.

We note that a cc with an orbital radius of $8.1 \mathrm{AU}$ was at the limit of the detectable zone in 2004 in the 4 QPM images, but as seen earlier, it is not possible from these data to definitively draw any firm conclusions on the detectability of a cc with $a \leq 30 \mathrm{AU}$ in 2004. We therefore regard the 8.1 AU solution as a possible one. A cc with an orbital radius of 17.1 AU was much too close to the star to be detected in 2004 or 2009 . Lecavelier des Etangs \& Vidal-Madjar (2009) proposed that a planet orbiting at 17.1 AU could have been detected when it was near quadrature 

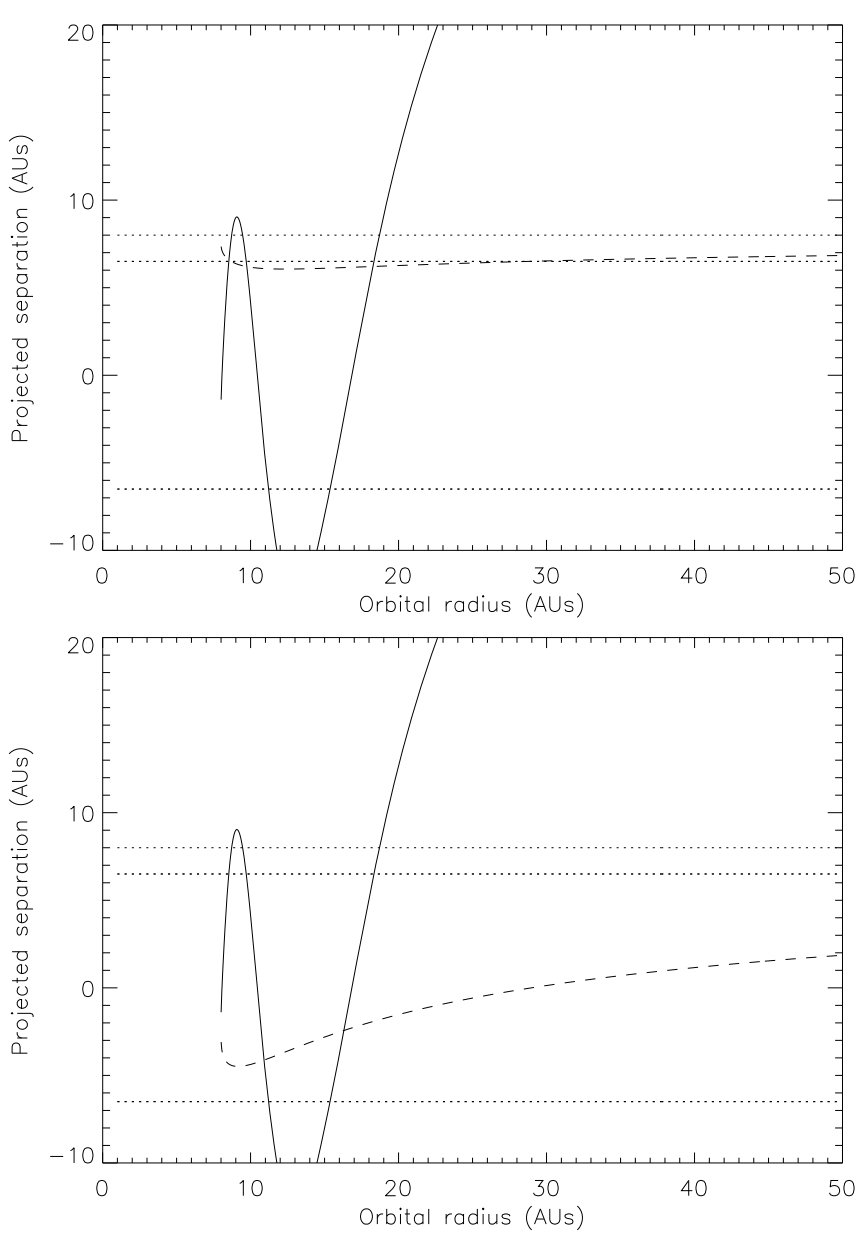

Fig. 6. Top: projected separation of the cc in 2004 (dashed curve) and 1981 (solid line curve), as a function of its orbital radius, assuming its projected separation was $8 \mathrm{AU}$ in 2003. Bottom: projected separation of the cc in 2009 (dashed curve) and in 1981 (solid line curve), as a function of its radius, assuming its projected separation was $8 \mathrm{AU}$ in 2003. In both cases, we took into account the fact that the cc was located after quadrature in November 2003. Horizontal lines: same conventions as in Fig. 5. Circular orbits are assumed and the $\mathrm{cc}$ is assumed to rotate prograde with respect to the circumstellar disk.

between 1993 and 1998, and thus excluded this solution. This is in fact not so clear, as the visible magnitude of the cc is estimated to be $V=21$, below the detection limits of the $1997 \mathrm{HST} / \mathrm{STIS}$ observations that probed the surroundings of $\beta$ Pic as close as 15 AU for companions with $V<17$ (Heap et al. 2000; Lagrange et al. 2009).

\subsection{Expected separations of the $c c$ in forthcoming years}

Given the constraints brought by the $L^{\prime}$ and 4QPM images, we plot in Fig. 8 the projected separation of the cc in the forthcoming years, assuming a circular orbit. We see that if its actual orbital radius is low (between 8 and 12-15 AU), the cc should be detectable again end of 2009, under good atmospheric conditions. If we take into account only the constraints provided by $L^{\prime}$-band data, we cannot exclude a cc before quadrature with an orbital radius smaller than 9.5 AU; in that case (see also Fig. 8), the cc would be detectable again only after 2012 .
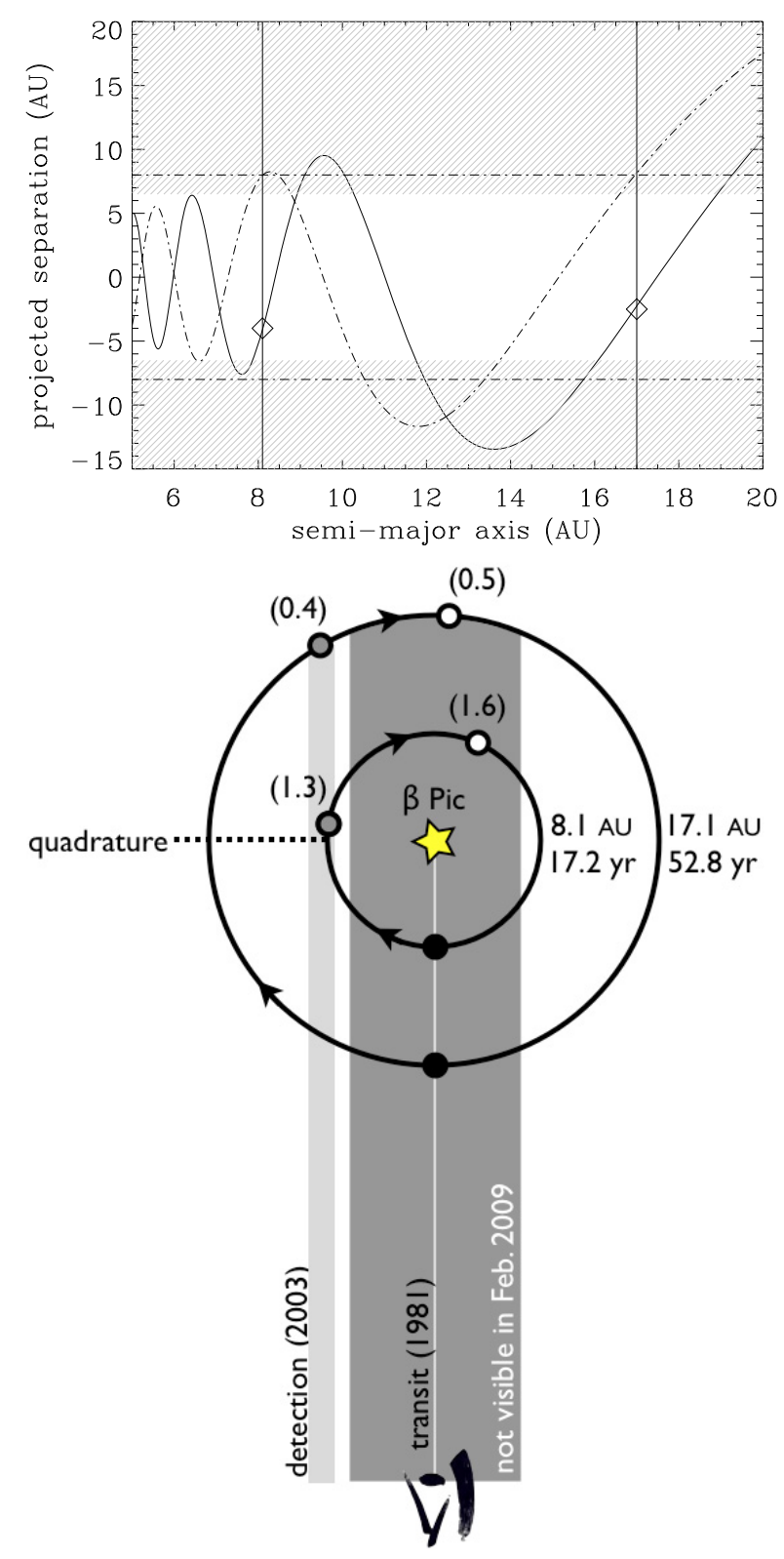

Fig. 7. Top: projected separation of the cc in 2003 (dash-dotted curve) and 2009 (solid curve) as a function of its orbital radius, assuming it was transiting $\beta$ Pic in 1981. The horizontal dash-dotted line represents the projected separation of the cc in 2003. Circular orbits are assumed, and the cc is assumed to rotate prograde with respect to the circumstellar disk. Bottom: sketch of the possible locations in November 2003 and February 2009 (as seen from above) of the cc assuming it transited $\beta$ Pic in 1981. The two cases $a=8.5$ and $a=17.1 \mathrm{AU}$ are represented (see text). The values in parenthesis give the fraction of the orbit that has been covered in 2003 (or 2009).

\section{Summary and future prospects}

New $L^{\prime}$-band data were obtained in February 2009 and did not reveal the presence of the cc detected in 2003 down to $6.5 \mathrm{AU}$. We show that the non detection does not allow us to rule out a background companion. The $L^{\prime}$-band data allow us to exclude initial positions for the cc before quadrature and with radii larger than 9.75 AU (circular orbits assumed). However, they do not constrain the possible orbits of a cc located after quadrature in 2003. 4QPM data obtained in January 2009 did not have the sensitivity to detect the $\mathrm{cc}$ in the $K \mathrm{~s}$ band. 

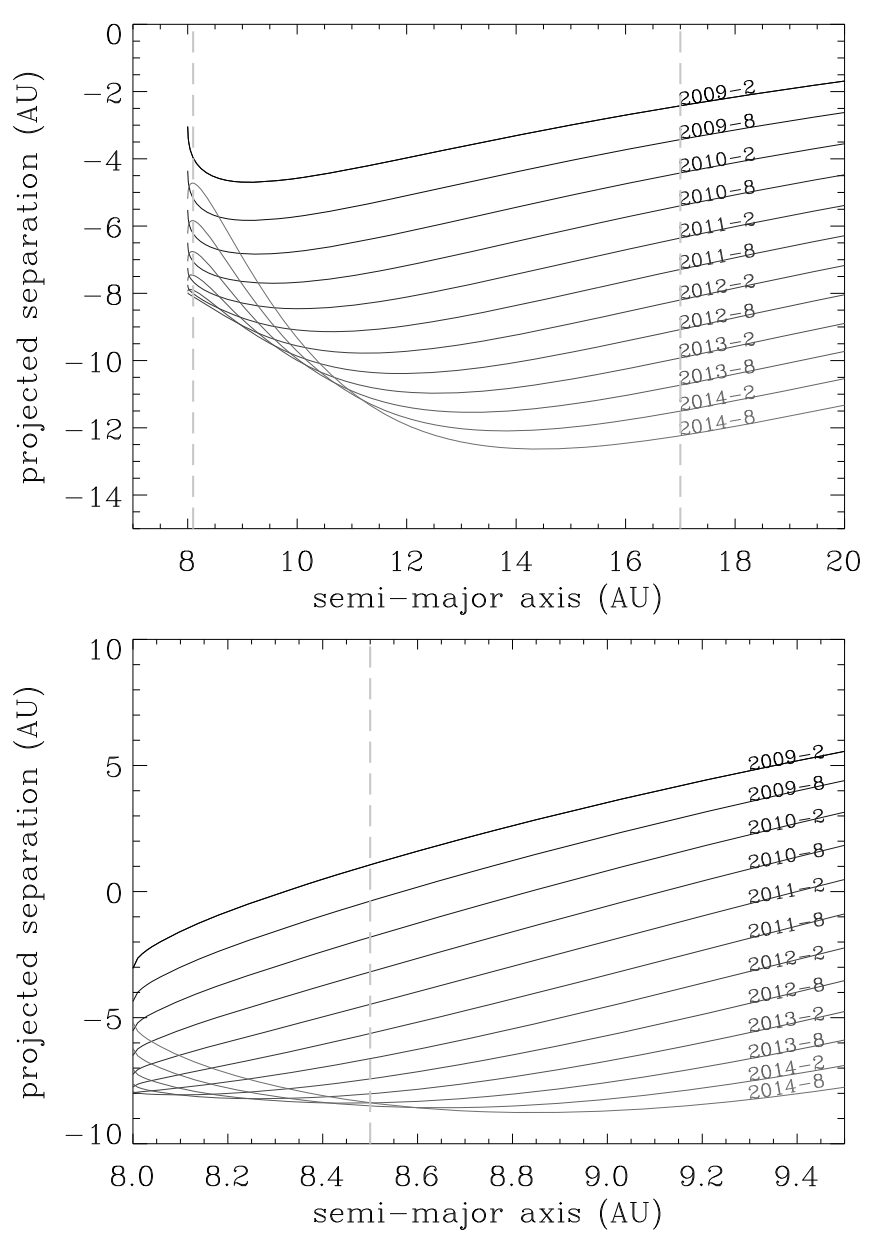

Fig. 8. Projected separation of the cc in forthcoming years as a function of its orbital radius, assuming its projected separation was $8 \mathrm{AU}$ in 2003. Left: we assume, as suggested by the $K$ s-band images, that the cc was located after quadrature in November 2003. Right: projected separation if the cc was observed before quadrature in November 2003. The vertical dashed lines at 8.5 and 17. AU indicate the possible (circular) separations if we further assume that the cc transited in 1981.

Assuming a $K s-L^{\prime}$ color of $1.2-1.5$, a similar analysis made on the 2004 4QPM data allowed to exclude us all initial configurations where the $\mathrm{cc}$ was located before quadrature. Moreover, they restrict the possible orbital radius of the cc to less than a maximum radius of $\simeq 30-100$ AU from the star. Nevertheless, the conclusions derived from the 4QPM data rely on $K \mathrm{~s}-L^{\prime}$ color estimations.

Finally, we have shown that if its actual orbital radius is small (between 8 and 12-15 AU), the cc could be detectable at $L^{\prime}$ as soon as end of 2009 under very good atmospheric conditions if after quadrature, and after 2012 if before quadrature.

Acknowledgements. We would like to thank ESO staff, in particular Lowell Tacconi for his very efficient support during phase II preparation, Paranal staff for the SM observations, and Christophe Dumas for his help. We thank Z. Haiman for pointing out precious information on galaxies/QSO contamination. We acknowledge financial support from the French Programme National de Planétologie (PNP, INSU), as well as from the French Agence Nationale pour la Recherche (ANR; project NT05 - 4_44463). These results have made use of the SIMBAD database, operated at CDS, Strasbourg, France.

\section{References}

Baraffe, I., Chabrier, G., Barman, T. S., Allard, F., \& Hauschildt, P. H. 2003, A\&A, 402, 701

Boccaletti, A, Baudrand, J., Baudoz, P., \& Riaud, P. 2007, in the Spirit of Bernard Lyot: The Direct Detection of Planets and Circumstellar Disks in the 21st Century, ed. P., Kalas, 39

Boccaletti, A., Chauvin, G., Baudoz, P., et al. 2008, A\&A, 482, 939

Boccaletti, A., Augereau, J.-C., Baudoz, P., et al. 2009, A\&A, 495, 523

Olofsson, G., Liseau, R., \& Brandeker, A. 2001, ApJ, 563, 770

Chabrier, G., Baraffe, I., Allard, F., \& Hauschildt, P. H. 2000, ApJ, 542, 464

Chauvin G., Lagrange A.-M., Dumas C., et al. 2005, A\&A, 438, L25

Chauvin G., Lagrange A.-M., Zuckerman B., et al. 2005, A\&A, 438, L29

Crifo, F., Vidal-Madjar, A., Lallement, R., Ferlet, R., \& Gerbaldi, M. 1997, A\&A, 320, L29

Haiman, Z., Spergel, D. N., \& Turner, E. L. 2003, ApJ, 585, 630

Kasper, M., Apai, D., Janson, M., \& Brandner, W. 2007, A\&A, 472, 321

Lafrenière, D., Doyon, R., Marois, C., et al. 2008, ApJ, 670, 1367

Lagrange, A. M., Gratadour, D., Chauvin, G., et al. 2009, A\&A, 493, L21

Lecavelier des Etangs, A., \& Vidal-Madjar, A. 2009, A\&A, 497, 557

Lecavelier des Etangs, A., Deleuil, M., Vidal-Madjar, A., et al. 1995, A\&A, 299, 557

Lecavelier des Etangs, A., Vidal-Madjar, A., Burki, G., et al. 1997, A\&A, 328, 311

Lenzen, R., Hartung, M., Brandner, W., et al. 2003, SPIE, 4841, 944

Marois, C., Lafreniere, D., Doyon, R., Macintosh, B., \& Nadeau, D. 2006, ApJ, 641,556

Marois, C., MacIntosh, B., Barman, T., et al. 2008, Science, 322, 1348

Rousset, G., Lacombe, F., Puget, P., et al. 2003, SPIE, 4839, 140

Zuckerman, B., Song, I., Bessel, M. S., \& Webb, R. A. 2001, ApJ, 562, L87 